\title{
Cooperative Wireless Medium Access Exploiting Multi-Beam Adaptive Arrays and Relay Selection
}

\author{
Xin Li, Member, IEEE, Yimin Zhang, Senior Member, IEEE, and Moeness G. Amin, Fellow, IEEE
}

\begin{abstract}
Cooperative transmission among wireless network nodes can be exploited to resolve collisions and thereby enhance the network throughput. Incorporation of multi-beam adaptive array (MBAA) at a base station/access point (destination) receiver has been shown to improve the network performance. In this paper, we propose an efficient cooperative wireless medium access scheme that exploits novel relay selection methods in a network equipped with MBAA at the destination receiver. Unlike existing techniques that require the estimation of angles-of-arrival (AoAs), the proposed scheme uses the spatial correlation among users for simpler but more effective collision detection and resolution. We present two useful relay selection methods based on channel gain and spatial correlation. It is shown that the joint use of an effective relay selection method and an MBAA in a wireless network can significantly improve the uplink throughput. The throughput of the proposed scheme and its upper bound are analytically derived. Numerical and simulation results have demonstrated significant performance enhancement achieved by the proposed cooperative wireless medium access scheme.
\end{abstract}

Index Terms-Cooperative network, collision resolution, multibeam adaptive array, relay selection, throughput analysis.

\section{INTRODUCTION}

$\mathbf{T}$ HE increasing demands for the delivery of multimedia contents over various wireless networks, such as cellular or wireless local area networks (WLANs), have motivated the development of new technologies that support higher network throughput. One of the trends is the use of flexible random access of the media resource, rather than traditional fixed channel allocations, to take advantage of the bursty nature of most multimedia sources [1]. Early random access protocols, such as ALOHA and slotted-ALOHA, offer a straightforward implementation but suffer from a severe throughput penalty and underutilization of the channel resources as the result of packet collision. Collision resolution mechanisms that retrieve collided packets are attractive to improve network throughput because of the effective utilization of these collided packets otherwise would be discarded (e.g., [2]-[4]).

Manuscript received December 16, 2008; accepted May 19, 2009. First published June 10, 2009; current version published October 14, 2009. The associate editor coordinating the review of this manuscript and approving it for publication was Prof. Alfred Hanssen. This work was supported in part by NSF under Grant EEC-0332490. Part of this work was presented at the IEEE International Conference on Circuits and Systems for Communications, Shanghai, China, May 2008, and at the IEEE International Conference on Acoustics, Speech, and Signal Processing, Taipei, Taiwan, April 2009.

The authors are with the Center for Advanced Communications, Villanova University, Villanova, PA 19085 USA e-mail: xin.1@villanova.edu; xinlivu@ yahoo.com; yimin.zhang@villanova.edu; moeness.amin@villanova. edu).

Digital Object Identifier 10.1109/TSP.2009.2025098
The investigation of collision resolution has been performed from both medium access control (MAC) as well as physical (PHY) layer perspectives. Earlier work assumes a single antenna at a base station or access point (destination) receiver, whereas the recent trend is to equip the destination receiver with multiple antennas so as to achieve spatial diversity. In [5], a single receive antenna scheme, referred to as network-assisted time diversity multiple access (NDMA), was proposed. Unlike traditional random access protocols which discard collided packets, the NDMA stores collided packets in memory and later combines them with retransmissions initiated during the following time slots. When the channel states change over different time slots such that the mixtures received at the receiver can be considered linearly independent, multiple sets of data mixtures can be stacked to form a model that resembles a multiple-input multiple-output (MIMO) system. Accordingly, the original data packets can be retrieved, provided that the equivalent MIMO channel matrix is full-column rank and can be estimated. To relax the assumption of fast time-varying channel, a modification was developed in [6], where channel coefficients are changed by additional weights in each retransmission. Nevertheless, this approach remains inefficient when the channels experience deep bursty fading.

The ALLow Improved Access in the Network via Cooperation and Energy Savings (ALLIANCES) scheme, developed in [7] and [8], achieves cooperative diversity in spatial and temporal domains and, as a result, it properly functions even in a slowly time-varying channel environment. In this scheme, collided packets are buffered and at least one cooperative transmission epoch (CTE) is triggered once collisions occur. In each CTE slot sequential to the collision slot, only one relay node, which can be either a source node or a nonsource node, is selected in turn to transmit. A source node retransmits the packet it already transmitted in the collision slot, whereas a nonsource node amplifies and forwards the mixed data packets it received in the collision slot. Similar to the NDMA, the ALLIANCES protocol also forms an equivalent MIMO problem to retrieve the original packets. This scheme maintains the benefits of slottedALOHA in the sense that all nodes share access to media resources. It is worth noting that a proper selection of the relay nodes, e.g., based on the location information of each node, can further improve the performance [8].

The ALLIANCES was then extended to exploit multi-beam adaptive arrays (MBAAs) so as to further improve throughput [9], [10]. An MBAA may separate multiple concurrent data packets without collision. Therefore, when an MBAA is used at the destination receiver, the arrival of data packets from multiple source nodes does not necessarily yield collision. CTE slots are 
triggered only when collision is considered to happen. In such an event, a reduced number of CTE slots suffices for the destination receiver to collect sufficient number of independent equations to retrieve the original data packets. In the ALLIANCES protocol that exploits MBAA [9], the detection of collision is based on the angular separation of the signal arrivals, and only the beams individually containing a single user are used in packet recovery. As is well known, with the use of an $M$-element array, the evaluation of angular separation based on angle-of-arrival (AoA) estimation methods (e.g., MUSIC [11] or ESPRIT [12]) can only resolve at most $M-1$, instead of $M$, users, yielding inefficient relay node utilization.

The objective of this paper is to enhance the network throughput by exploiting an efficient cooperative wireless medium access scheme to be utilized at a destination receiver equipped with an MBAA [10], and to provide throughput with an analytical framework. Two effective relay selection methods are proposed to further improve the throughput performance. The main contributions of this paper are summarized as follows. First, different from the existing techniques [7]-[9], we utilize all the information collected both in the collision slot and CTE slot(s) to jointly retrieve data packets at the final CTE slot. The cooperative diversity can thus be fully achieved. Second, the detection of the occurrence of packet collisions in a beam is eliminated. As such, the necessity of AoA estimation is removed. The maximum possible number of independent equations to be formed at each time slot is increased up to $M$ instead of $M-1$ that was achieved in [9]. Third, we use the generalized spatial signature vector, which can be derived from channel estimation, to describe the spatial characteristics of the signal from a node. Thus, the proposed scheme can provide a more accurate description of the channel states in a multipath propagation environment compared to the schemes based on the estimation of AoA information. Fourth, the proposed relay selection methods make better use of the channel coefficients at the destination receiver to properly select the relay nodes. The first proposed relay selection method selects relay nodes based on the channel coefficient magnitude, whereas the other method further enforces low spatial correlation between the selected channels. The two methods improve the probability of having high channel gains and reduce the dependence among channels so as to ensure that the channel matrix reaches full column-rank with the smallest number of time slots. As a result, the number of CTE slots can be significantly reduced and the uplink throughput is subsequently improved. Furthermore, we provide analytical evaluation and the upper bound of the throughput performance. It is shown through analytical and simulation results that a typical throughput enhancement of about $25 \%$ to $50 \%$ can be achieved in a network with moderate to high traffic load. The throughput improvement is further enhanced when the proposed relay selection methods are utilized in a network of large size.

The rest of the paper is organized as follows. Section II provides the network assumptions. In Section III, the enhanced cooperative medium access protocol with MBAA is described and mathematically formulated. Two relay selection methods are presented in Section IV. The throughput performance is analytically studied in Section V. Simulation results are provided in Section VI. Finally, we conclude this paper in Section VII.

\section{NETWORK ASSUMPTIONS}

For the underlying network system, we make the following assumptions similar to those introduced in [7].

1) Consider a slotted multiple access network system, where all the $J$ nodes are synchronized to the destination receiver. Both the link delay and decoding delay at the destination receiver are ignored.

2) All nodes operate in a half-duplex mode (either transmit or receive at a time) and use the same carrier frequency channel. A node not in the transmit state stays in the receive state, and thus it can receive packets from other nodes.

3) Each node can hear the messages from the destination receiver on a downlink control channel and is thus informed, at each time slot, whether it should transmit a source packet, forward a cooperative packet, or stay in a receive status.

4) The destination receiver is equipped with an $M$-element adaptive array whereas only one antenna is equipped at each node.

5) A unique user ID is inserted at the beginning of each uplink source data packet. The length of the data packet is the same as that of one time slot.

6) Channel coefficients remain constant within a time slot such that array processing at the destination receiver is feasible. Consider that channels are slowly time-varying or quasi-static during cooperative transmission. Thus, channel coefficients at the collision slot can be applicable to the subsequent CTE slots.

The emphasis of this paper is on the uplink throughput enhancement by using the efficient cooperative access scheme. The throughput is thus defined as the expected value of the number of source nodes from which packets are successfully received in a time slot [5]-[10], [13]-[17]. For example, if the successful reception of data packets transmitted from $K$ source nodes requires a mean number of $\mathcal{E}(\hat{K} \mid K)$ time slots, where $\mathcal{E}(\cdot)$ denotes the expectation operator, the throughput corresponding to $K$-user scenario is then expressed as $(K) /(\mathcal{E}(\hat{K} \mid K))$. The reason of using the uplink throughput rather than uplink capacity as the performance measure is that it is often infeasible to achieve uplink capacity by merely adjusting data rates of source or cooperative nodes in the underlying network. Moreover, a relay node does not have the flexibility to change the data rate of relay, because the data rate is determined by the upstream from the source nodes and a cooperative node does not decode the packets it forwards.

\section{Cooperative Medium Access Protocol}

\section{A. General Description}

It is assumed that in each CTE time slot, one or several nodes can act as relays to cooperate the packet transmission via the wireless channels. Due to the application of MBAA at the destination receiver, the spatial dimensionality of channels can be effectively utilized for the provision of robust and high throughput wireless links. Suppose that the destination receiver is able to concurrently receive signals from up to $M$ nodes without collision. Packet collision occurs and CTE slots are initialized only when the channel matrix is rank deficient, which happens either 
when the number of active users exceeds the receiver capacity or when the channels are highly correlated. That is, a CTE slot may not be required if the number of simultaneous users $K$ satisfies $K \leq M$, whereas the packets collide and at least one CTE slot is needed when $K>M$. Note that the value of $K$ can be estimated using active user detection techniques [5], [7], and [9]. At each CTE slot, $M_{r} \geq 1$ relay nodes are selected to simultaneously transmit. A relay node can be either a source node or a cooperative (nonsource) node. When a source node is selected, it retransmits its own packet previously transmitted at the collision slot. When a nonsource node is selected, it amplifies and forwards the signal mixture it received during the collision slot. In either case, due to the use of array signal processing techniques, the cooperative transmission at each CTE slot can contribute up to $\min \left(M, M_{r}\right)$ independent equations which are to be stacked to resolve the collided packets in a faster and better fashion.

\section{B. Signal Model}

Consider that $K$ active source users transmit their respective data packets at the $n$th time slot (namely, non-CTE slot or collision slot). Depending on the channel states and the number of active users $(K)$, the $K$ data packets may collide and thus cannot be retrieved based on the data received at the $n$th time slot alone. Let $S(n)=\left\{i_{1}, \ldots, i_{K}\right\}$ denote the set of active source nodes, $\bar{S}(n)=\left\{r_{1}, \ldots, r_{\bar{K}}\right\}$ be that of the $\bar{K}=J-$ $K$ nonsource nodes, and $D=\left\{d_{1}, \ldots, d_{M}\right\}$ contain the $M$ MBAA antennas at the destination receiver. Assume that the packet sent by the $i_{k}$ th node consists of $N$ symbols $\mathbf{x}_{i_{k}}(n)=$ $\left[x_{i_{k}, 0}(n), \ldots, x_{i_{k}, N-1}(n)\right]$, where $k=1, \ldots, K$. The received signal at an MBAA antenna or a nonsource node is then given by the $1 \times N$ row vector

$$
\mathbf{y}_{c}(n)=\sum_{i_{k} \in S(n)} h_{c i_{k}}(n) \mathbf{x}_{i_{k}}+\mathbf{n}_{c}(n)
$$

where $c \in D \bigcup \bar{S}(n)$ denotes an antenna at the destination receiver or a nonsource relay node (referred to as the $c$ th sensor hereafter), $h_{c i_{k}}$ is the channel coefficient from the $i_{k}$ th source node to the $c$ th sensor, and $\mathbf{n}_{c}(n) \in \mathbb{C}^{1 \times N}$ is the additive white Gaussian noise (AWGN) vector with zero mean and variance $\sigma_{n}^{2}$. The channel coefficients from the nodes to the destination receiver are assumed to be slowly time-varying normalized independent and identically distributed (i.i.d.) complex Gaussian variables with zero mean and unit variance. The channel coefficients among the nodes are assumed to be quasi-static and realized from i.i.d. complex Gaussian variables with zero mean and unit variance. Note that these assumptions are not necessary to the proposed scheme, but will facilitate the performance analysis and simulations. For convenience, the signal received by the MBAA at the $n$th slot can be written in form of the $M \times N$ matrix

$$
\begin{aligned}
\mathbf{Y}(n) & =\left[\mathbf{y}_{d_{1}}^{T}(n), \ldots, \mathbf{y}_{d_{M}}^{T}(n)\right]^{T} \\
& =\mathbf{H}(n) \mathbf{X}(n)+\mathbf{n}(n)
\end{aligned}
$$

where $\mathbf{y}_{d_{j}}(n)$ is given in (1), the channel matrix $\mathbf{H}(n)=$ $\left[\mathbf{h}_{D i_{1}}(n), \ldots, \mathbf{h}_{D i_{K}}(n)\right] \in \mathbb{C}^{M \times K}$ with $\mathbf{h}_{D i_{k}}(n)=$ $\left[h_{d_{1} i_{k}}(n), \ldots, h_{d_{M} i_{k}}(n)\right]^{T}, \mathbf{X}(n)=\left[\mathbf{x}_{i_{1}}^{T}(n), \ldots, \mathbf{x}_{i_{K}}^{T}(n)\right]^{T} \in$
$\mathbb{C}^{K \times N}, \mathbf{n}(n)=\left[\mathbf{n}_{1}(n), \ldots, \mathbf{n}_{M}(n)\right]^{T} \in \mathbb{C}^{M \times N}$ is the noise matrix, and $(\cdot)^{T}$ denotes the transpose of a matrix or a vector. Each channel coefficient can be estimated, for example, through the orthogonal ID sequence that is attached to the beginning of the packet [5], [7]. Consequently, $M$ linear equations are constructed.

\section{Existing Data Retrieving Technique}

There are different ways for a cooperative scheme to retrieve each collided packet. The decoding method used in [9] is to decode packets at both the non-CTE slot and the final CTE time slot in two steps. At the non-CTE slot, the AoAs of active users are first estimated via the ESPRIT algorithm and the existence of packet collision is identified. The packets free of beam overlapping are then retrieved with beamforming method. In the event that some active users have close AoAs, the corresponding packets are considered to be collided. Subsequently, the CTE procedure is initiated to resolve the remaining collided packets. At each CTE slot, $M_{r} \leq M-1$ relay nodes are selected in turn for cooperative transmission. The CTE continues until enough independent equations are gathered to resolve the collided packets. Only the packets free of collision are used for collision resolution, whereas others are discarded.

The performance of the above data retrieving technique may be impeded for several reasons. The collision detection based on AoA information is generally not effective because angle separation is not always a good measure to identify the spatial dependence between two nodes. For example, two well-separated nodes may have high spatial dependence if one is close to the direction of the other's grating lobe. On the other hand, when multipath propagation occurs, channels cannot be precisely described solely by the AoAs. Compared to AoA-based approaches, spatial signature-based collision detection is considered more reliable. In addition, collision resolution based only on the packets free of collision is inefficient, as the collided packets with close but different spatial signatures can contribute to collision resolution as well. Consequently, joint packet retrieving performed only at the final CTE slot using all the available received data is more effective than proceeding with two separate steps. This is discussed below.

\section{Proposed Data Retrieving Technique}

In the proposed technique, packet collision is not explicitly detected at each time slot. The destination receiver only identifies whether the (stacked) channel matrix is full-column rank. The receiver does not retrieve packets if the channel matrix is column rank-deficient. When the channel matrix $\mathbf{H}(n)$ is fullcolumn rank, the array system at the destination receiver is able to resolve the data packets, and thus a CTE slot is not triggered. On the other hand, when this condition does not hold, e.g., in case $K>M, \mathbf{H}(n)$ becomes column rank-deficient and thus the $K$ packets cannot be resolved at this time slot. The CTE procedure is subsequently initiated so as to resolve all collided packets via cooperative transmission. If there are $\hat{K}-1 \mathrm{CTE}$ slots following the $n$th time slot so that the $K$ data packets can be retrieved, the total number of $\hat{K}$ time slots are thus required to cooperatively deliver the $K$ data packets. Once the stacked channel matrix becomes full-column rank, the CTE procedure 
is terminated, and all the collided packets are jointly retrieved at the end of the final CTE slot. Regardless whether packets collide or not collide at each time slot, all the received data packets are used in the recovery of data. In the following, the proposed packet retrieving method is described in detail.

Assume that at the $t$ th time slot (i.e., the $(t-n)$ th CTE slot), where $n<t \leq(n+\hat{K}-1)$, there are $q$ nonsource relay nodes that are selected to forward the $K$ packets that they individually received at the $n$th time slot, and there are $p=M_{r}-q$ source nodes retransmitting their own packets. The signal received by the MBAA in this time slot can also be written in form of an $M \times N$ matrix

$$
\begin{aligned}
\mathbf{Y}(t) & =\left[\mathbf{y}_{d_{1}}^{T}(t), \ldots, \mathbf{y}_{d_{M}}^{T}(t)\right]^{T} \\
& =\left[\mathbf{H}_{\mathbf{s}}(t)+\mathbf{H}_{\mathbf{r}}(t)\right] \mathbf{X}(n)+\mathbf{n}(t)
\end{aligned}
$$

where

$$
\begin{aligned}
\mathbf{H}_{\mathbf{s}}(t) & =\mathbf{H}(t) \mathbf{P}^{(p)}(t) \\
\mathbf{H}_{\mathbf{r}}(t) & =\sum_{j=1}^{q} \gamma_{r_{j}}(t) \mathbf{h}_{D r_{j}}(t) \mathbf{h}_{r_{j} S}^{T}(t) \\
& =\mathbf{H}_{D R}(t) \mathbf{Q}^{(q)}(t) \mathbf{H}_{R S}(t) \\
\mathbf{n}(t) & =\sum_{j=1}^{q} \gamma_{r_{j}}(t) \mathbf{h}_{D r_{j}}(t) \mathbf{n}_{r_{j}}(t)+\mathbf{n}_{D}(t) \\
& =\mathbf{H}_{D R}(t) \mathbf{Q}^{(q)}(t) \mathbf{n}_{D R}(t)+\mathbf{n}_{D}(t) .
\end{aligned}
$$

The parameters in the above equations are described below.

- $\mathbf{H}_{\mathbf{s}}(t) \in \mathbb{C}^{M \times K}$ is the channel matrix denoting the uplink channel coefficients from the $p$ source relay nodes to the destination receiver at the $(t-n)$ th CTE time slot.

- $\mathbf{P}^{(p)}(t) \in \mathbb{R}^{K \times K}$ is a diagonal matrix whose diagonal entries corresponding to the $p$ selected source relay nodes are 1's and others are 0's.

- $\mathbf{H}_{\mathbf{r}}(t) \in \mathbb{C}^{M \times K}$ is the combined channel matrix consisting of both the channels $\mathbf{h}_{r_{j} S}(t) \in \mathbb{C}^{K \times 1}$, representing channel coefficients from all source nodes to each of the $q$ nonsource relay nodes, and the channels $\mathbf{h}_{D r_{j}}(t) \in \mathbb{C}^{M \times 1}$, denoting channel coefficients from each nonsource relay node to the destination receiver, $j=1, \ldots, q$.

- $\mathbf{H}_{D R}(t)=\left[\cdots, \mathbf{h}_{D r_{j}}(t), \cdots\right] \in \mathbb{C}^{M \times \bar{K}}$ is the channel matrix denoting the uplink channel coefficients from all $\bar{K}$ nonsource relay nodes to the destination. Note that $\mathbf{H}_{D R}(t)$ is a subset of $\mathbf{H}(t)$.

- $\mathbf{H}_{R S}(t)=\left[\cdots, \mathbf{h}_{r_{j} S}(t), \cdots\right]^{T} \in \mathbb{C}^{\bar{K} \times K}$ is the channel matrix representing the channel coefficients from all $K$ source relay nodes to the $\bar{K}$ nonsource relay nodes.

- $\mathbf{Q}^{(q)}(t) \in \mathbb{R}^{\bar{K} \times \bar{K}}$ is a diagonal matrix whose entries corresponding to the $q$ selected nonsource relay nodes are relay scaling factors $\gamma_{r_{j}}$ and others are 0's. The scaling factor $\gamma_{r_{j}}$ at the $j$ th relay node is defined as

$$
\gamma_{r_{j}}(t)=\sqrt{\frac{\sigma_{x}^{2}}{\left\|\mathbf{h}_{r_{j} S}(t)\right\|^{2} \sigma_{x}^{2}+\sigma_{n}^{2}}}
$$

where $\sigma_{x}^{2}$ is the average power of transmitted symbols.
- $\mathbf{n}(t) \in \mathbb{C}^{M \times N}$ is the combined noise matrix, consisting of both the noise $\mathbf{n}_{D}(t)$ observed at the receiver array and the relay noise. The latter is the AWGN $\mathbf{n}_{D R}(t) \in$ $\mathbb{C}^{\bar{K} \times N}$ observed at the nonsource relay nodes and then delivered to the destination receiver via the fading propagation channel after scaling and amplifications at the nonsource relay nodes. All elements in $\mathbf{n}_{D}(t)$ as well as in $\mathbf{n}_{r_{j}}(t), j=1, \ldots, q$, are assumed to be of zero mean and variance $\sigma_{n}^{2}$.

Following the procedure described above, the stacked signal received at the destination receiver during the $n$th time slot and the subsequent $\hat{K}-1$ CTE time slots can be expressed as

$$
\mathbf{Y}=\mathbf{H X}(n)+\mathbf{n}
$$

where

$$
\begin{aligned}
& \mathbf{Y}=\left[\mathbf{Y}(n)^{T}, \mathbf{Y}(n+1)^{T}, \ldots, \mathbf{Y}(n+\hat{K}-1)^{T}\right]^{T} \in \mathbb{C}^{M \hat{K} \times N} \\
& \mathbf{H}=\left[\begin{array}{c}
\mathbf{H}(n) \\
\mathbf{H}_{\mathbf{s}}(n+1)+\mathbf{H}_{\mathbf{r}}(n+1) \\
\vdots \\
\mathbf{H}_{\mathbf{s}}(n+\hat{K}-1)+\mathbf{H}_{\mathbf{r}}(n+\hat{K}-1)
\end{array}\right] \in \mathbb{C}^{M \hat{K} \times K}(10) \\
& \mathbf{n}=\left[\mathbf{n}(n)^{T}, \mathbf{n}(n+1)^{T}, \ldots, \mathbf{n}(n+\hat{K}-1)^{T}\right]^{T} \in \mathbb{C}^{M \hat{K} \times N} .
\end{aligned}
$$

It is worth noting that the destination receiver is not required to separately estimate channels $\mathbf{H}_{\mathbf{s}}(t)$ and $\mathbf{H}_{\mathbf{r}}(t)$ for $t=n+$ $1, \ldots,(n+\hat{K}-1)$, contained in (10) and respectively given in (4) and (5). The estimation of their combination suffices for the purpose of packet retrieving. Once the stacked channel matrix $\mathbf{H}$ reaches full-column rank, the CTE procedure is terminated, and all the data packets originated from the $K$ users can be retrieved through a proper multi-user detection technique. Optimally, the collided packets are decoded using a maximum-likelihood (ML) decoder, which minimizes the following Frobenius norm with respect to $\mathbf{X}(n)$ under the set space $\Omega$ of all possible transmitted symbols, i.e.,

$$
\hat{\mathbf{X}}(n)=\underset{\mathbf{X}(n) \in \Omega}{\arg \min }\|\mathbf{Y}-\mathbf{H X}(n)\| .
$$

The ML approach can fully exploit the cooperative diversity provided by the system and achieve optimal performance at the expense of high decoding complexity. Several suboptimal decoding approaches with lower computation cost are also available. For example, the zero-forcing (ZF) linear decoder can be utilized and the retrieved data packets are expressed as

$$
\hat{\mathbf{X}}(n)=\mathbf{H}^{\dagger} \mathbf{Y}
$$

where $\mathbf{H}^{\dagger}$ is the pseudo-inverse of $\mathbf{H}$.

As discussed above, in contrast to the decoding approach used in [9], the proposed method above can efficiently retrieve the collided packets and thus reduce the total number of required time slots. As a consequence, the throughput can be significantly enhanced. 


\section{RElay SELECTION METHODS}

In the cooperative medium access scheme proposed in the previous section, $M_{r}$ relay nodes are selected to forward or retransmit at each CTE slot, providing $M_{r}$ additional linear equations. Since each node may experience different fading and propagation attenuation, an elaborate relay node selection method can further improve the throughput, as an improved relay selection method yields full-rank channel matrix with a smaller number of CTE slots through proper configuration of $\mathbf{P}^{(p)}$ in (4) and $\mathbf{Q}^{(q)}$ in (5). In this section, we consider all nodes are distributed in a similar environment and closely spaced, and two relay selection methods are proposed [10]. In the following, we first consider the channel estimation which is essential to efficient selections of relay nodes.

\section{A. Channel Estimation}

As discussed in Section III, there are two kinds of channels: one is the uplink channel $\left(\mathbf{H}_{\mathbf{S}}\right)$ from source nodes to the destination receiver, the other is the channel $\left(\mathbf{H}_{R S}\right)$ in between nodes. Note that, as given in (5), channel $\mathbf{H}_{\mathbf{r}}$ is the combined version of the two kinds of channels. To estimate these channels, additional overhead could be imposed on the content of a data packet, for example, by inserting an orthogonal ID sequence into the packet. Thus, channel coefficients can be estimated by performing a simple correlation operation at the receiver [5], [7]. Meanwhile, the detection of active users at the non-CTE slot can still be achieved using a similar operation. Therefore, the advantage of inserting an orthogonal ID sequence is to accomplish both accurate channel estimation and active user detection at the same time. Slowly time-varying channels are assumed, i.e., channel coefficients obtained at the non-CTE time slot remain unchanged at the subsequent CTE slots. Therefore, the additional burden for channel estimation is acceptable considering the advantages achieved from the application of the proposed cooperative access scheme, particularly when the length of data packets is relatively long.

\section{B. Relay Selection Based on Channel Gain}

It is observed in (6) that each packet received at the destination receiver may contain two kinds of noise, i.e., AWGN and relay noise originated from nonsource relay nodes. From (3), the received signal at the destination receiver can be strengthened by exploiting high-gain channels $\mathbf{H}_{\mathbf{s}}$ and $\mathbf{H}_{\mathbf{r}}$. According to (6), the relay noise is proportional to $\mathbf{H}_{D R}$, whereas the AWGN $\mathbf{n}_{D}$ is not impacted by the channel gain. When all these channels are available, we can select the nodes with the highest channel gain so as to make the system robust and immuned to both sources of noise. However, the estimation of channel coefficients $\mathbf{H}_{R S}(n)$ from the source nodes to nonsource relay nodes is impractical as the relay nodes usually cannot afford such capability. Instead, we consider the node selection based only on the uplink channel coefficients $\left(\mathbf{H}(n)\right.$ and $\left.\mathbf{H}_{D R}(n)\right)$ from node $n_{k} \in\{S(n) \bigcup \bar{S}(n)\}$ to the destination receiver, denoted as $\mathbf{h}_{D n_{k}}(n), k=1, \ldots, J$. As the consequence, the AWGN $\mathbf{n}_{D}$ is mitigated and uplink throughput can be enhanced. To this end, we provide the following relay selection algorithm.
Before starting the first CTE slot, a list of relay nodes is formed by sorting the uplink channels in the descend order of their gain values as

$$
\mathbf{L}(n)=\left\{n_{1}, \ldots, n_{J}\right\}
$$

where the condition $\left\|\mathbf{h}_{D n_{1}}(n)\right\| \geq \cdots \geq\left\|\mathbf{h}_{D n_{J}}(n)\right\|$ is satisfied. The $M_{r}$ relay nodes at the $t$ th slot $(t>n)$ are then taken from $\mathbf{L}(n)$ and the relay node list $\mathbf{L}_{t}$ is

$$
\mathbf{L}_{t}=\left\{n_{\bmod \left(M_{r}(t-1-n), J\right)+1}, \ldots, n_{\bmod \left(M_{r}(t-n)-1, J\right)+1}\right\}
$$

where function $\bmod (\cdot)$ denotes the modulus after division. With the use of relay selection above, the channel matrix $\mathbf{H}$ given in (9) can reach full-column rank in a faster manner.

\section{Relay Selection Based on Channel Gain and Spatial Correlation}

An alternative and more effective approach is to utilize both channel gain and spatial correlation of the packet signals for the selection of relay nodes. The beams formed by the MBAA can be optimized for the reception of a packet originated from a node. The interference from other nodes depends on the closeness of their spatial signatures, described using the spatial correlation function. When the channel coefficients corresponding to nodes $i$ and $j$ are denoted as $\mathbf{h}_{D i}$ and $\mathbf{h}_{D j}$, respectively, their spatial correlation coefficient is defined as

$$
\rho_{i j}=\frac{\mathbf{h}_{D i}^{H} \mathbf{h}_{D j}}{\left\|\mathbf{h}_{D i}\right\|\left\|\mathbf{h}_{D j}\right\|}, \quad i, j=1, \ldots, J .
$$

A low magnitude of the spatial correlation coefficient between two signals implies low interference and thus improves the output signal-to-interference-plus-noise ratio (SINR) [16], [18]. The selection of $M_{r}$ relay nodes with low spatial correlation therein in each CTE slot decreases the likelihood of packet collisions in the spatial dimension, and thus the number of CTE slots required for collision resolution can be reduced. As mentioned earlier, high-gain channels can mitigate AWGN at the receiver. Consequently, a more effective approach for relay selection is to jointly consider the channel gain as well as the spatial correlation.

Denote $\rho_{0}$ as the threshold of spatial correlation coefficient, i.e., the maximum correlation coefficient magnitude between two channels to be considered of low correlation. To construct the relay node list $\mathbf{L}_{t}=\left\{n_{1}^{t}, \ldots, n_{M_{r}}^{t}\right\}$ at the $t$ th slot under the consideration of both channel gain and spatial correlation, we develop the algorithm for the generation of relay nodes, and the flowchart is illustrated in Fig. 1, where the set $\mathbf{U}$ consists of already selected relay nodes in all elapsed CTE slots, $\Phi$ denotes the empty set, and the function $\operatorname{setdiff}(A, B)$ takes the set difference: $A-B$. Moreover, some comments are given below.

C1) If collision occurs at the $n$th slot and the CTE procedure is initiated, the destination receiver first estimates the uplink channel coefficients $\mathbf{h}_{D n_{k}}(n)(k=1, \ldots, J)$ and then forms the relay list $\mathbf{L}(n)$ given in (14). 


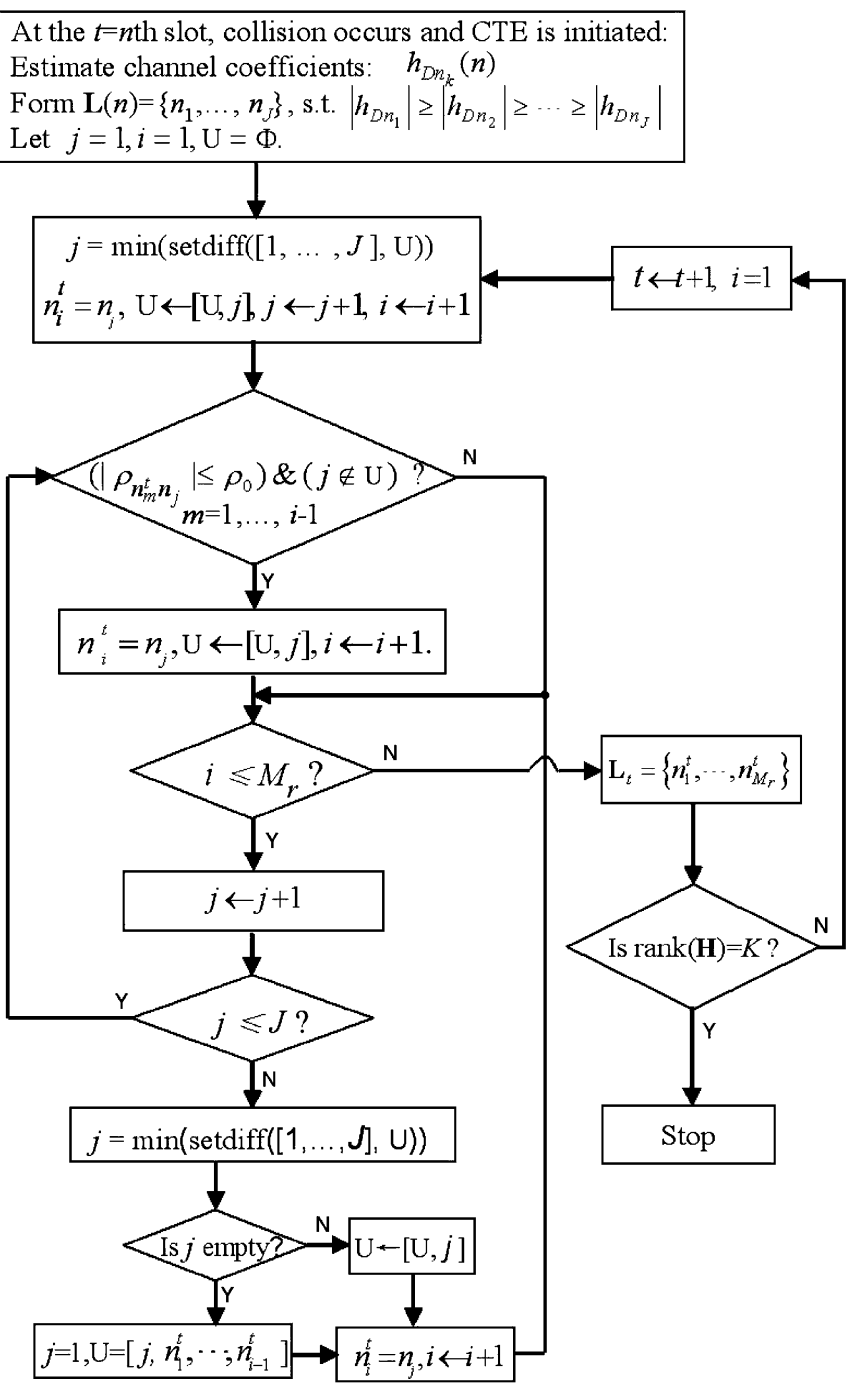

Fig. 1. Flowchart of the algorithm for the generation of relay nodes under the consideration of both channel gain and spatial correlation.

C2) Parameter $i$ equals the number of already selected relay nodes at the current CTE slot, and $j$ is the location pointer toward the candidate of relay nodes under consideration.

C3) By default, a new relay node is selected only if its spatial correlation with all preselected relay nodes is lower than $\rho_{0}$. If such a relay does not exist, the node with the highest channel gain is selected from the unselected nodes to fill up the deficiency. In the case of nonexistence of such a node (i.e., every qualified node is already selected to act as relay), the node with the highest channel gain is selected, and the set $\mathbf{U}$ is reset and refilled with the already selected nodes in the current CTE slot.

C4) When $M_{r}$ relay nodes are selected, the destination receiver updates the stacked channel matrix $\mathbf{H}$ given in (9). The CTE procedure is terminated if $\mathbf{H}$ becomes full-column rank, otherwise another CTE slot is proceeded.

Remarks: i) The algorithm performance is sensitive to the preset value of $\rho_{0}$. The relay selection method is gradually de- generated to the selection method based on high gain channels as $\rho_{0}$ takes a high value (i.e., $\left|\rho_{0}\right| \rightarrow 1$ ). ii) It is assumed that each node in the network can act as a relay. In the event of a nonfully connected network, a candidate relay set consisting of only the nodes that are able to relay at the CTE slots can be generated, and the updated version of this algorithm can be obtained in a straightforward way. iii) Because the stop criterion for the equation collection during the CTE is the full-column rank of the channel matrix, the full-column rank condition for the stacked channel matrix is another important factor. A loose condition seems to reduce the number of CTE slots and thus to amplify the throughput, but it may result in more decoding errors and thereby degenerate the throughput. On the other hand, a strict condition may lead to the collection of redundant equations and prolonged duration of the CTE and thus it also decreases the throughput. Therefore, the condition of determining the full-column rank must be well selected. This is to be further discussed in the next section.

\section{PERFORMANCE ANALYSIS}

In this section, we analyze the throughput performance of the cooperative wireless medium access scheme in forms of upper bound and accurate evaluation [29].

\section{A. Generic Results}

Without loss of generality, the uplink throughput $G$, as mentioned in Section II, is defined as the expected value of the simultaneous throughput gain $(K / \hat{K})$, i.e., the average number of nodes from which packets are successfully received in a time slot [5]-[10], [13]-[17].

For clarity, it is assumed that all $J$ nodes are randomly located in the network area with an independent uniform angular distribution around the destination receiver and each node acts as a source node to transmit a packet with an identical probability $p_{s}$, though the case of the nonidentical probability and dependent queuing is possible and discussed in [8], [19], [20]. The probability that, at the $n$th time slot, $K$ out of $J$ nodes actively transmit source packets is then given by

$$
P(K)=\left(\begin{array}{c}
J \\
K
\end{array}\right) p_{s}^{K}\left(1-p_{s}\right)^{J-K}
$$

where $\left(\begin{array}{c}J \\ K\end{array}\right)$ denotes the combination operation representing the number of different ways of selecting $K$ out of $J$ nodes.

Let $P(\hat{K} \mid K)$ be the conditional probability that exact $\hat{K}$ slots are required to resolve packet collisions in the presence of $K$ active nodes at the $n$th time slot. Clearly, for a given $K$, the value of $\hat{K}$ can be any positive integer. Thus, the expected value of $\hat{K}$ in this case is

$$
\mathcal{E}(\hat{K} \mid K)=\sum_{\hat{K}=1}^{\infty} \hat{K} P(\hat{K} \mid K) .
$$

Consequently, the conditional throughput in the presence of $K$ active nodes can be written as

$$
G_{K}=\frac{K}{\mathcal{E}(\hat{K} \mid K)}
$$


Eventually, the uplink throughput is given by

$$
G=\mathcal{E}\left(G_{K}\right)=\sum_{K=1}^{J} G_{K} P(K) .
$$

In the following, we analyze the uplink throughput in two manners: upper bound and accurate evaluation. The most important part of the analysis is the derivation of the conditional probability $P(\hat{K} \mid K)$.

\section{B. Upper Bound}

First, we analyze the upper bound of the uplink throughput, which is derived based on the maximum possible number of independent equations the received data can offer. Toward this end, the channel fading and noise effects are ignored. In the presence of $K$ active users, the expected minimum number of time slots required by collision resolution can be expressed as

$$
\mathcal{E}(\hat{K} \mid K)^{\mathrm{ub}}=\left\lceil\frac{K}{M}\right\rceil
$$

where $\lceil x\rceil$ denotes the ceiling function whose value is the smallest integer not less than $x$. Therefore, the upper bound of the throughput in the presence of $K$ active nodes is

$$
G_{K}^{\mathrm{ub}}=\frac{K}{\mathcal{E}(\hat{K} \mid K)^{\mathrm{ub}}} .
$$

Subsequently, the upper bound of the uplink throughput is achieved as

$$
\begin{aligned}
G^{\mathrm{ub}} & =\sum_{K=1}^{J} G_{K}^{\mathrm{ub}} P(K) \\
& =\sum_{K=1}^{J} \frac{K}{\left\lceil\frac{K}{M}\right\rceil}\left(\begin{array}{c}
J \\
K
\end{array}\right) p_{s}^{K}\left(1-p_{s}\right)^{J-K} .
\end{aligned}
$$

This upper bound shows that it increases with both the number of array elements $M$ and the transmission probability $p_{s}$ of each node.

\section{Accurate Evaluation}

Next, we analyze the accurate evaluation of the throughput. From the protocol described in Section III, the event that the minimum number of time slots required for collision resolution in the presence of $K$ active nodes exactly equals $\hat{K}$ is found to be equivalent to the event that the stacked channel matrix $\mathbf{H}$, given in (10), is full-column rank. Therefore, their occurrence probabilities are also equal, i.e.,

$$
P(\hat{K} \mid K)=\operatorname{Pr}(\operatorname{rank}(\mathbf{H})=K)
$$

It is well known that the rank of a matrix equals the number of nonzero singular values. Thus, for $M \hat{K} \geq K, \mathbf{H}$ is full-column rank if and only if all its $K$ singular values are nonzero. Equivalently, $\mathbf{H}$ is full-column rank when its smallest singular value $\sigma_{\min }$ is nonzero. Practically, the channel matrix $\mathbf{H}$ can be considered full-column rank if $\sigma_{\min } \geq \sigma_{\text {th }}$, where $\sigma_{\text {th }}$ is a preset threshold. Thus, (24) can be written as

$$
P(\hat{K} \mid K)=\operatorname{Pr}\left(\sigma_{\min } \geq \sigma_{\mathrm{th}}\right)
$$

which is relevant to the problem of probability distribution of the smallest singular value of a matrix [21]-[27]. In the following, we discuss the problem according to the characteristics of the channel matrix.

The stacked channel matrix $\mathbf{H}$ is generally a complex Gaussian matrix whose elements are not necessarily i.i.d. We first consider the special case that $\mathbf{H} \sim \mathcal{C N}\left(\mathbf{0}, a^{2} \mathbf{I}_{K}\right)$, where $a$ is a scalar. Following the derivation in [22], the probability distribution of the smallest singular value of $\mathbf{H}$ is obtained as

$$
\operatorname{Pr}\left(\sigma_{\min } \geq \sigma_{\mathrm{th}}\right)=\left|\frac{\operatorname{det}\left(\mathbf{A}_{K, K_{d}, \sigma_{\mathrm{th}}^{2} / a^{2}}\right)}{\operatorname{det}\left(\mathbf{A}_{K, K_{d}, 0}\right)}\right|
$$

where $\operatorname{det}(\cdot)$ denotes the determinant of a matrix, and $K_{d}=$ $M \hat{K}-K$. Additionally, matrix $\mathbf{A}_{K, K_{d}, b} \in \mathbb{R}^{K \times K}$ is given by

$$
\mathbf{A}_{K, K_{d}, b}=\left[\begin{array}{ccc}
\tilde{\Gamma}_{b}\left(K_{d}+1\right) & \ldots & \tilde{\Gamma}_{b}\left(K_{d}+K\right) \\
\tilde{\Gamma}_{b}\left(K_{d}+2\right) & \cdots & \tilde{\Gamma}_{b}\left(K_{d}+K+1\right) \\
\vdots & & \vdots \\
\tilde{\Gamma}_{b}\left(K_{d}+K\right) & \cdots & \tilde{\Gamma}_{b}\left(K_{d}+2 K-1\right)
\end{array}\right]
$$

where

$$
\tilde{\Gamma}_{b}(x)=\int_{b}^{\infty} t^{x-1} e^{-t} d t=\Gamma(x)\left[1-\Gamma_{b}(x)\right] .
$$

In the above expression,

$$
\Gamma(x)=\int_{0}^{\infty} t^{x-1} e^{-t} d t
$$

is the Gamma function, and

$$
\Gamma_{b}(x)=\frac{1}{\Gamma(x)} \int_{0}^{b} t^{x-1} e^{-t} d t .
$$

is the incomplete Gamma function. Consequently, the probability distribution of the smallest singular value becomes tractable for a channel matrix with complex i.i.d. entries.

In general, the channel matrix is expressed as $\mathbf{H} \sim$ $\mathcal{C N}(\mathbf{0}, \boldsymbol{\Sigma})$, where the covariance matrix $\boldsymbol{\Sigma} \in \mathbb{C}^{K \times K}$ is not an identity matrix. In this case, we consider $\mathbf{W}=\mathbf{H}^{H} \mathbf{H}$, which is the complex central Wishart matrix with $K$ degrees of freedom [22]-[27]. From the distribution of the smallest eigenvalue $\lambda_{\min }$ of $\mathbf{W}$, the distribution of the smallest singular value of $\mathbf{H}$ can be derived as [25]

$$
\begin{aligned}
& \operatorname{Pr}\left(\sigma_{\min } \geq \sigma_{\mathrm{th}}\right) \\
& \quad=\operatorname{Pr}\left(\lambda_{\min } \geq \sigma_{\mathrm{th}}^{2}\right) \\
& \quad=\operatorname{etr}\left(-\sigma_{\mathrm{th}}^{2} \Sigma^{-1}\right) \sum_{k=0}^{K(M \hat{K}-K)} \widehat{\sum_{\mathcal{K}}} \frac{\mathcal{C}_{\mathcal{K}}\left(\sigma_{\mathrm{th}}^{2} \Sigma^{-1}\right)}{k !}
\end{aligned}
$$


where $\operatorname{etr}(\cdot)$ denotes the exponential of the trace, $\widehat{\sum_{\mathcal{K}}}$ represents the summation over the partitions ${ }^{1} \mathcal{K}=\left(k_{1}, \ldots, k_{K}\right)$ of $k$ with $k_{1} \leq(M \hat{K}-K)$, and $\mathcal{C}_{\mathcal{K}}(\mathbf{B})$ is the complex zonal polynomial of the complex matrix $\mathbf{B} \in \mathbb{C}^{K \times K}, \mathbf{B} \neq \mathbf{I}_{K}$, defined as [25], [26]

$$
\mathcal{C}_{\mathcal{K}}(\mathbf{B})=\chi_{[\mathcal{K}]}(\mathbf{1}) \cdot \chi_{[\mathcal{K}]}(\mathbf{B})
$$

with

$$
\begin{aligned}
\chi_{[\mathcal{K}]}(1) & =k ! \frac{\prod_{i<j}^{K}\left(k_{i}-k_{j}-i+j\right)}{\prod_{i=1}^{K}\left(k_{i}+K-i\right) !} \\
\chi_{[\mathcal{K}]}(\mathbf{B}) & =\frac{\operatorname{det}\left(\mathbf{A}_{n}\right)}{\operatorname{det}\left(\mathbf{A}_{d}\right)} .
\end{aligned}
$$

In the above equations, $\mathbf{A}_{n}$ and $\mathbf{A}_{d}$ have the same size of $K \times K$, and their $(i, j)$ th entries are respectively expressed as $\left(\mathbf{A}_{n}\right)_{i j}=$ $\lambda_{i}^{k_{j}+K-j}$ and $\left(\mathbf{A}_{d}\right)_{i j}=\lambda_{i}^{K-j}$, with $\lambda_{1}, \ldots, \lambda_{K}$ denoting the $K$ eigenvalues of B. Particularly, $\mathcal{C}_{\mathcal{K}}(x)=x^{k}$ for $K=1$ [26], and $\mathcal{C}_{\mathcal{K}}(\alpha \mathbf{B})=\alpha^{k} \mathcal{C}_{\mathcal{K}}(\mathbf{B})$ [23], where both $x$ and $\alpha$ are scalar variables.

When either $\boldsymbol{\Sigma}=a^{2} \mathbf{I}$ or $\mathbf{B}=\alpha \mathbf{I}$, (32) and (34) become invalid. Instead, (26) is just applicable in this case, or the following expression derived from the results given in [25], [26] can be used

$$
\mathcal{C}_{\mathcal{K}}\left(\alpha \mathbf{I}_{K}\right)=(4 \alpha)^{k} k !\left[\frac{K}{2}\right]_{\mathcal{K}} \frac{\prod_{i<j}^{r}\left(2 k_{i}-2 k_{j}-i+j\right)}{\prod_{i=1}^{r}\left(2 k_{i}+r-i\right) !}
$$

where

$$
\left[\frac{K}{2}\right]_{\mathcal{K}}=\prod_{i=1}^{r} \frac{\Gamma\left[(K-i+1) / 2+k_{i}\right]}{\Gamma[(K-i+1) / 2]}
$$

and $r$ is the number of nonzero parts in the partition $\mathcal{K}$ of $k$.

The above derivations provide the probability distribution of the smallest singular value of the channel matrix $\mathbf{H}$, i.e., the probability of $\mathbf{H}$ being full-column rank, provided that the channel matrix $\mathbf{H}$ is a complex Gaussian matrix with zero-mean entries. As a result, the closed-form expression of the accurate uplink throughput can be eventually written as

$$
G=\sum_{K=1}^{J} \frac{K\left(\begin{array}{c}
J \\
K
\end{array}\right) p_{s}^{K}\left(1-p_{s}\right)^{J-K}}{\sum_{\hat{K}=1}^{\infty} \hat{K} \operatorname{Pr}\left(\sigma_{\min } \geq \sigma_{\text {th }}\right)}
$$

where $\operatorname{Pr}\left(\sigma_{\min } \geq \sigma_{\text {th }}\right)$ is given in (31) for a general case of $\mathbf{H}$ except when $\bar{\Sigma}=a^{2} \mathbf{I}$ or $\mathbf{B}=\alpha \mathbf{I}$ is satisfied. In this case, $\operatorname{Pr}\left(\sigma_{\min } \geq \sigma_{\mathrm{th}}\right)$ can be obtained from (26) or (35).

\section{Discussions}

The generic throughput result described above provides the principle of throughput evaluation. The result of optimal throughput is presented in the form of upper bound, since it is obtained by considering the contributions of array gain/diversity but ignoring the impacts of channel fading and noise. Therefore, the upper bound of the throughput can be approached only in

\footnotetext{
${ }^{1}$ For the partition $\mathcal{K}=\left(k_{1}, \ldots, k_{K}\right)$ of the integer $k$, two conditions are
} satisfied: (1) $k_{1} \geq \cdots \geq k_{K} \geq 0$, and (2) $k=k_{1}+\cdots+k_{K}$. the high signal-to-noise ratio (SNR) regime. For the result of accurate evaluation, it is derived by taking consideration of channel characteristics other than the traffic load, where the stacked channels are approximated as complex Gaussian. It is also worth noting that the evaluated throughput can be impacted by the value of the preset threshold of the smallest singular value $\sigma_{\mathrm{th}}$. As is well known, at a high SNR, the channel matrix $\mathbf{H}$ can be accurately estimated and thus the estimation of the smallest singular value $\sigma_{\min }$ does not noticeably suffer detriment from noise. As a result, both accurate estimation of the probability of $\mathbf{H}$ being full-column rank and the robust evaluation of throughput performance are feasible. On the other hand, the estimated $\sigma_{\min }$ could be inaccurate due to noise perturbation in the low SNR regime, and thus the accuracy of the throughput evaluation is compromised. Consequently, the threshold $\sigma_{\text {th }}$ needs to be adjusted with the noise level so as to provide a robust throughput evaluation. As a rule of thumb, the threshold $\sigma_{\text {th }}$ can be roughly preset as

$$
\sigma_{\text {th }} \propto \frac{1}{\mathrm{SNR}}
$$

where the SNR is defined as the ratio of transmit power at the source nodes to the receiver noise power.

\section{Simulations}

In this section, simulations are carried out to demonstrate the performance of the cooperative medium access protocol and the relay selection methods. The effectiveness of the analytical performance analysis is also illustrated [29].

\section{A. Assumptions}

For fair comparison, we establish a simulation environment similar to that used in [5], [7] and [9]. Two configurations of the network size are considered. One has a relatively small size with $J=16$ nodes, and the other has a moderate size with $J=32$ nodes. The nodes' ID sequences are selected from a Hadamard matrix of order $J$. A Bernoulli model is used to generate active nodes at a certain traffic load $\lambda$, defined as the average number of active nodes at a non-CTE time slot. Each node independently transmits its packet with probability $p_{s}=\lambda / J$ at each non-CTE slot. The data packet length is fixed as $N=424$ bits (equal to the length of an ATM cell) and the BPSK modulation is used. The bit rate is $6 \mathrm{Mb} / \mathrm{s}$. Both ML and ZF decoding approaches are applied at the destination receiver. A packet retrieved with bit error rate (BER) of $P_{e}=0.01$ or higher is considered corrupted and thus it is not counted in the evaluation of throughput. The input SNR of 10 or $15 \mathrm{~dB}$ at each receive antenna is considered. 1000 repeated independent non-CTE slots are emulated at each traffic load to compute the uplink throughput.

Due to the orthogonality of packet ID sequences, the accuracy of detection of active users is satisfactory in most situations, even in the relatively low SNR regime. In our simulations, perfect detection of active users during the non-CTE slot is assumed so as to focus on the performance evaluation of the collision resolution. It is considered that the destination receiver is equipped with a uniform linear array (ULA) consisting of $M=4$ elements with a half-wavelength interelement spacing. The AoAs 


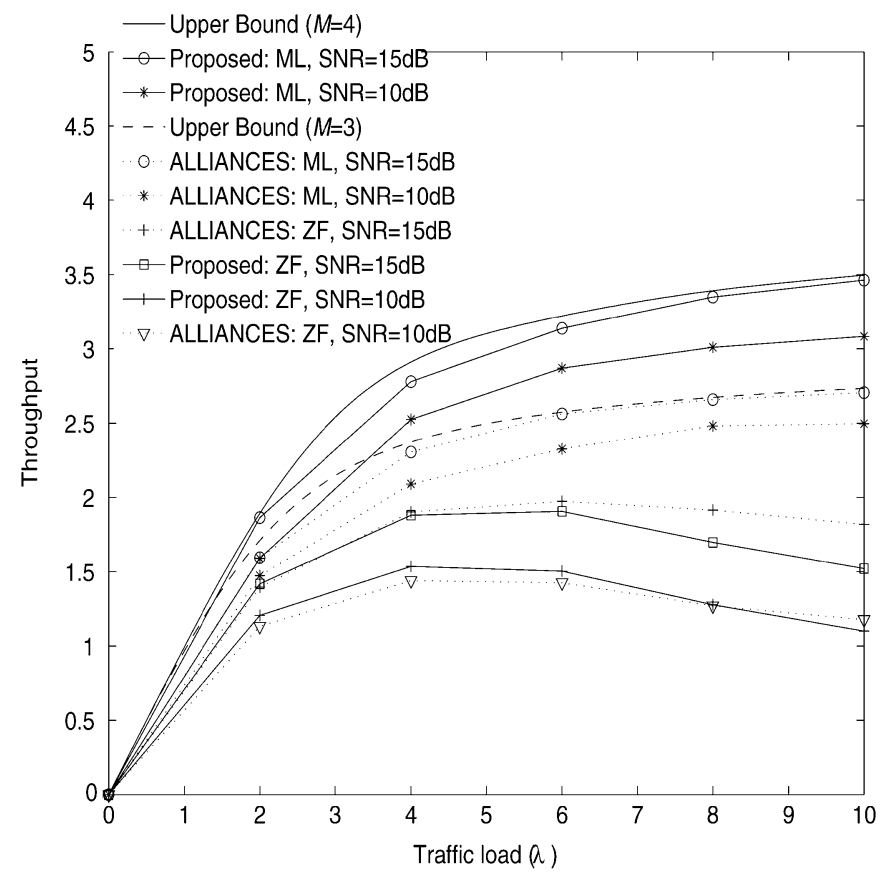

Fig. 2. Throughput comparison among proposed scheme and the upper bound of ALLIANCES with relay selection in turn $\left(J=16, \sigma_{\mathrm{th}}=0.1\right.$ for SNR $=$ $10 \mathrm{~dB}, \sigma_{\mathrm{th}}=0.01$ for SNR $=15 \mathrm{~dB}$ ).

of the nodes at the destination receiver are randomly distributed with a uniform angular distribution. The channel from each node to the destination receiver is faded with an i.i.d. Rayleigh distribution and is simulated using the Jakes' model [7], [28]. The carrier frequency is $5.2 \mathrm{GHz}$, and the maximum doppler frequency shift is $f_{d}=52 \mathrm{~Hz}$, corresponding to a vehicle speed of $v=3 \mathrm{~m} / \mathrm{s}$. Note that the channels among nodes are assumed to be quasi-static complex Gaussian variables, since most nodes of interest in the network are usually located in a similar environment and closely spaced, and thus are slowly time-varying.

\section{B. Comparison of Throughput Performance}

In [9], only those beams that individually contain a single packet in each time slot are used to retrieve the packets. Due to the use of AoA estimation, the maximum number of available beams is confined to at most $M-1$ in all the time slots. In our proposed scheme described in Section III, we can exploit all the $M$ beams formed in both the non-CTE and CTE slots to retrieve data packets. As a result, up to $M$ relay nodes are used at each CTE slot. Clearly, the throughput performance of the proposed scheme is improved when a proper decoding approach, such as the ML decoding described in Section III, is used. The comparison between the throughput performance of the proposed scheme and the upper bound of ALLIANCES 2 with relay selection in turn is illustrated in Fig. 2, where both ML and ZF decoding approaches are used $(J=16)$. Two input SNR values of 10 and $15 \mathrm{~dB}$ are respectively considered, and the corresponding threshold values $\sigma_{\mathrm{th}} 0.1$ and 0.01 are assumed. The upper bound curves of our proposed scheme are calculated based on (23). It is observed in Fig. 2 that the throughput of the

\footnotetext{
${ }^{2}$ The throughput upper bound of ALLIANCES is approximately obtained under the following assumptions. The detection of active users is assumed to be perfect. The number of beams free of collision is confined to $M-1$ and all the received packets are used for collision resolution at the final CTE slot.
}

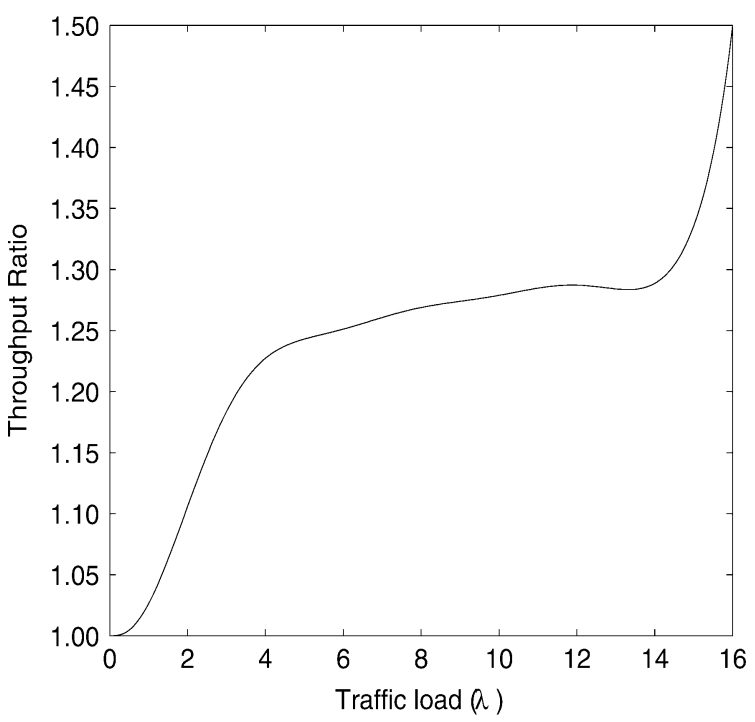

Fig. 3. Throughput ratio between proposed scheme and the upper bound of ALLIANCES with relay selection in turn $(J=16, M=4)$.

proposed scheme supporting $M$ relay nodes provides significant improvement when the ML decoding approach is applied. When the ZF decoding approach is used, on the other hand, the throughput is improved only when the traffic load is under saturate. As described in Section V, the throughput approaches the upper bound when the input SNR is sufficiently high. It is interesting to note that the performance of throughput upper bound of ALLIANCES is nearly equivalent to that of the proposed scheme with a $(M-1)$-element array. Consequently, in the high SNR regime, the throughput ratio $R_{G}$ of the proposed scheme to the upper bound of ALLIANCES can be written as

$$
R_{G}=G^{\mathrm{ub}}(M) / G^{\mathrm{ub}}(M-1)
$$

where $G^{\mathrm{ub}}(\cdot)$ is given in (23). The numeric results are plotted in Fig. 3. Clearly, the throughput improvement of the proposed scheme becomes more significant as the traffic load is higher. For example, the throughput increment is about $25 \%$ at moderate load and such an increment is up to $50 \%$ when the traffic load is 16. In contrast, such throughput enhancement over the available throughput of ALLIANCES [9] is even higher.

\section{Impact of $\sigma_{\mathrm{th}}$ and Decoding Methods}

As discussed in Section $\mathrm{V}$, the evaluated throughput is impacted by the value of the preset threshold of the smallest singular value $\sigma_{\text {th }}$. Here, we examine such an impact. The conditional upper bound under $K$ active nodes is given in (22). The conditional throughput versus the number of active nodes under different threshold values is illustrated in Fig. 4, where $J=16$ and SNR $=10 \mathrm{~dB}$ are assumed. It is observed that, for a given value of input SNR, a lower value of $\sigma_{\text {th }}$ leads to a higher rank-based throughput since, under a looser decision condition, fewer CTE slots are required to make the channel matrix full-column rank. Accordingly, an efficient decoding method is required to asymptotically achieve such a higher throughput. Instead, the throughput is compromised if a relatively poor decoding method, such as $\mathrm{ZF}$, is used. This has been shown in Fig. 2, where the use of ZF decoding approach leads to throughput penalty, especially when the traffic load 


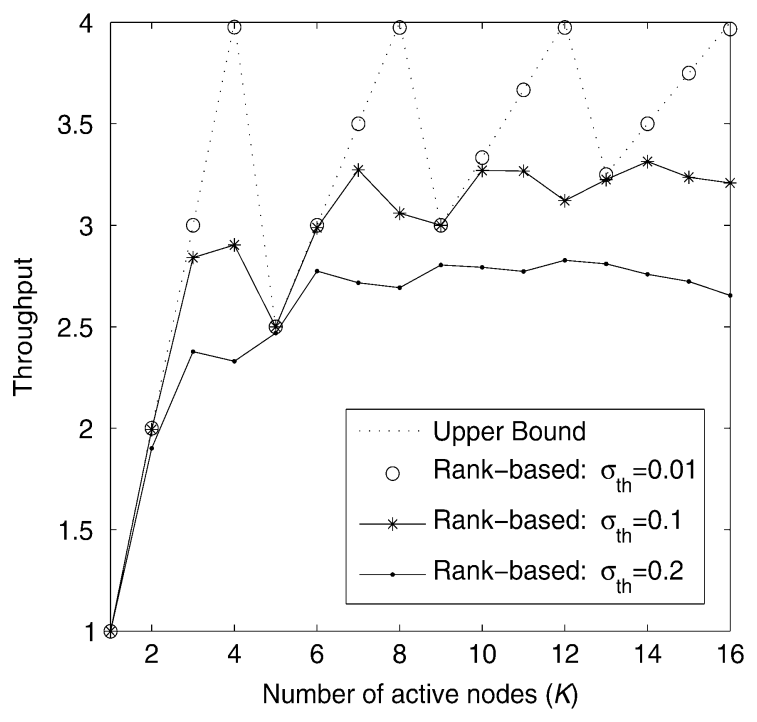

Fig. 4. Conditional throughput versus the number of active nodes under different threshold values $\sigma_{\text {th }}$ with relay selection in turn $(J=16$, SNR $=$ $10 \mathrm{~dB})$.

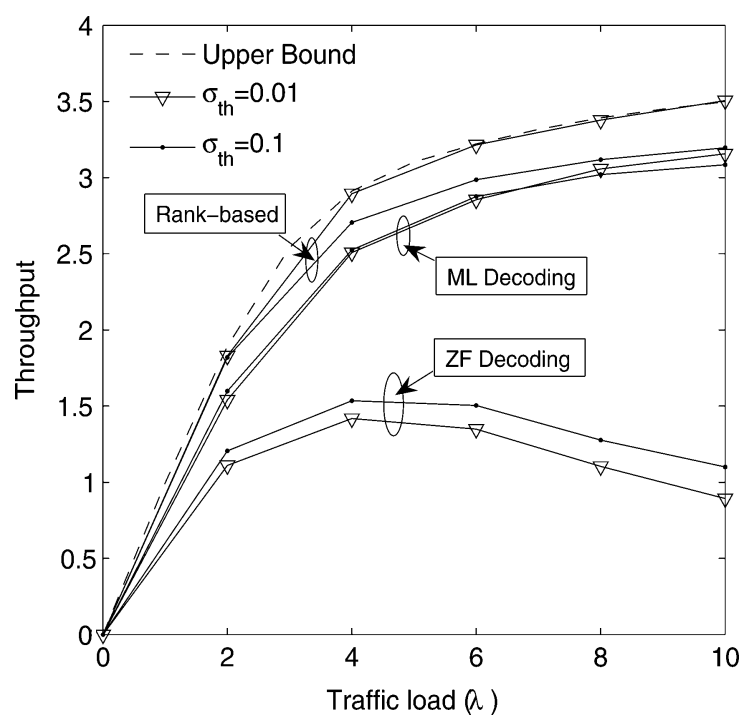

Fig. 5. Throughput comparison under both different threshold values $\sigma_{\text {th }}$ and decoding methods with relay selection in turn $(J=16, \mathrm{SNR}=10 \mathrm{~dB})$.

is oversaturate. This phenomenon is further demonstrated in Fig. 5, where $J=16$ and SNR $=10 \mathrm{~dB}$ are assumed. When the ML decoding scheme is applied, the achieved throughput at $\sigma_{\mathrm{th}}=0.01$ is nearly identical to that obtained at $\sigma_{\mathrm{th}}=0.1$. However, when the ZF decoding is used, a low threshold $\sigma_{\mathrm{th}}=0.01$ yields poor throughput performance. This is because that the ZF receiver, as expressed in (13), may cause noise enhancement and thus result in more decoding errors and lower throughput. By contrast, the ML receiver, as expressed in (12), does not have such a problem and thus achieves a higher throughput. Furthermore, comparing the throughput results obtained from ML decoding as shown in Fig. 2 for SNR = $15 \mathrm{~dB}$ and $\sigma_{\mathrm{th}}=0.01$ (the solid line with cycle mark) and in Fig. 5 for SNR $=10 \mathrm{~dB}$ and $\sigma_{\mathrm{th}}=0.1$ (solid line with dot mark), it is observed that at a high SNR, a low value of $\sigma_{\mathrm{th}}$ is preferable. This coincides with the discussion in Section V.

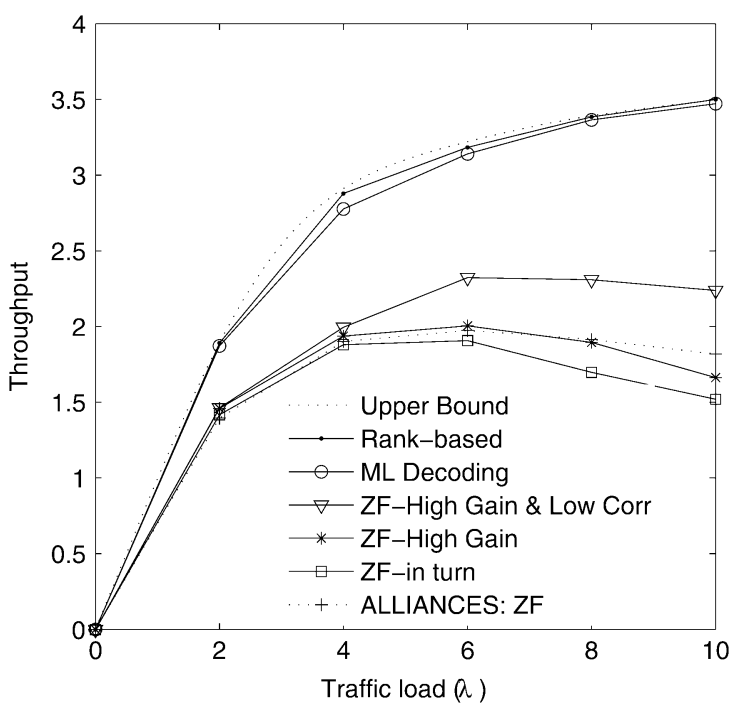

Fig. 6. Throughput comparison of the proposed scheme with various relay selections $\left(J=16, \mathrm{SNR}=15 \mathrm{~dB}, \sigma_{\mathrm{th}}=0.01\right)$.

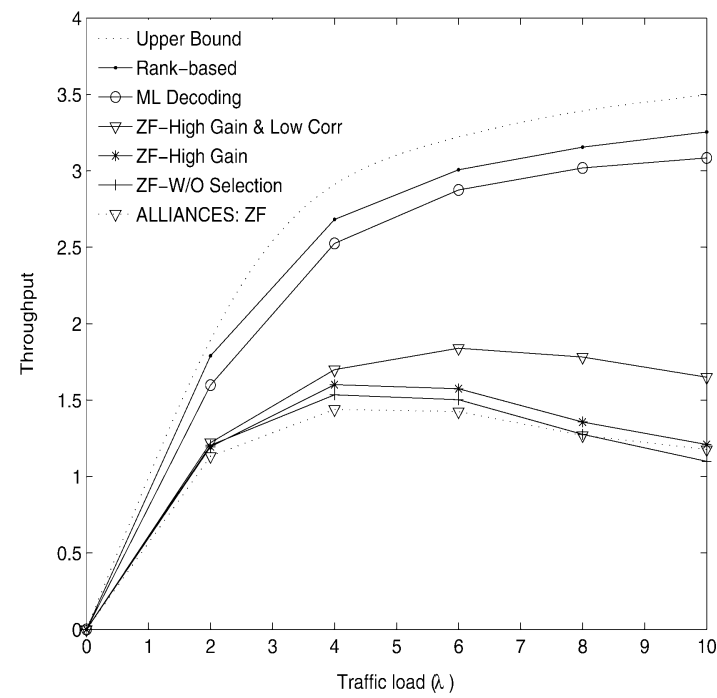

Fig. 7. Throughput comparison of the proposed scheme with various relay selections $\left(J=16, \mathrm{SNR}=10 \mathrm{~dB}, \sigma_{\mathrm{th}}=0.1\right)$.

\section{Effectiveness of Relay Selection Methods}

The throughput performance of the proposed scheme with various relay selection methods is compared and the results are illustrated in Fig. 6 for the case of SNR $=15 \mathrm{~dB}$ and $\sigma_{\text {th }}=0.01$ and in Fig. 7 for the case of SNR $=10 \mathrm{~dB}$ and $\sigma_{\text {th }}=0.1$. A relatively small-scale network consisting of $J=16$ nodes is assumed. In each simulation, the ZF decoding approach is first used due to its low computation load and the ML approach is then applied. When the spatial correlation is considered, the correlation threshold is set to $\rho_{0}=0.5$. The results show that, when the relay nodes are selected based only on the channel gain, the throughput improvement over the case where relay nodes are chosen in turn [9] is not significant. However, significant throughput gain is achieved when the relay nodes are selected based on both channel gain and the spatial correlation. More importantly, both figures show that at a relatively high 


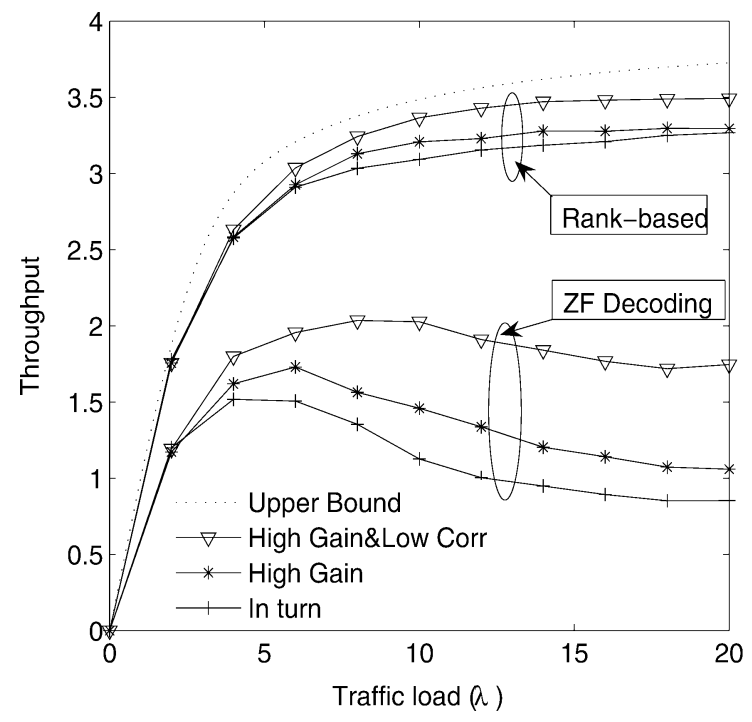

Fig. 8. Throughput comparison of the proposed scheme with various relay selections $\left(J=32, \mathrm{SNR}=10 \mathrm{~dB}, \sigma_{\mathrm{th}}=0.1\right)$.

traffic load, such throughput improvement becomes more pronounced, since the selection method takes advantage of more efficient use of the spatial dimensionality. It is worth noting that, different from the results in Fig. 2 where relay nodes are selected in turn, when the relay selection approach is taken into account, the throughput improvement of the proposed scheme over ALLIANCES, even under the ZF decoding, becomes significant. It is not surprising that when the ML decoding approach is applied, the throughput curves, which are not illustrated for clarity, are more approaching the rank-based results.

\section{E. Impact of Network Size}

For comparison, we further consider a network of larger size, where $J=32$. The throughput of the proposed scheme with various relay selections is shown in Fig. 8, where $\mathrm{SNR}=10 \mathrm{~dB}$ and $\sigma_{\mathrm{th}}=0.1$ are assumed. Comparing the results illustrated in Fig. 7 for $J=16$ and Fig. 8 for $J=32$, it is observed that the throughput is improved. Clearly, the throughput improvement due to the use of the proposed relay selection methods is more significant in a larger-scale network, because a network of larger size is likely to provide more preferable relay candidates, and thus more efficient use of the spatial dimensionality can be achieved.

\section{CONCLUSION}

In this paper, we have proposed an efficient cooperative wireless medium access scheme exploiting novel relay selection techniques in a network equipped with multi-beam adaptive array (MBAA) at the destination receiver. In the proposed scheme, the spatial signature of the received signal, instead of angle-of-arrivals (AoAs) or location-based information, is used for the effective collision detection and resolution. As such, the proposed technique eliminates the complexity of AoA estimation and the necessity of knowing node location information in advance. The spatial dimension of MBAA is fully utilized in the proposed techniques to resolve collisions and enhance throughput. Furthermore, we developed two efficient relay selection methods based on channel gain and spatial correlation for the optimal use of the spatial dimensionality. In addition, we analyzed the throughput performance of the proposed scheme and provided an analytical upper bound that approximates the actual throughput performance in the high SNR regime. Accurate evaluation of the throughput performance was achieved using the probability distribution of the smallest singular value of the channel matrix. Extensive simulation results validated the attractive performance of the proposed cooperative wireless medium access scheme. As the result, the proposed techniques significantly improve the uplink throughput, particularly for a network of relatively large size.

\section{REFERENCES}

[1] A. S. Tanenbaum, Computer Networks, 3rd ed. Upper Saddle River, NJ: Prentice-Hall, 1996.

[2] Q. Zhao and L. Tong, "Semi-blind collision resolution in random-access wireless ad hoc network," IEEE Trans. Signal Process., vol. 48, no. 10, pp. 2910-2920, Oct. 2000.

[3] X. Li, "Contention resolution in random-access wireless networks based on orthogonal complementary codes," IEEE Trans. Commun., vol. 52, no. 1, pp. 82-89, Jan. 2004.

[4] J. Wang, Y. Fang, and D. Wu, "Enhancing the performance of medium access control for WLANs with multi-beam access point," IEEE Trans. Wireless Commun., vol. 6, no. 2, pp. 556-565, Feb. 2007.

[5] M. K. Tsatsanis, R. Zhang, and S. Banerjee, "Network-assisted diversity for random access wireless networks," IEEE Trans. Signal Process., vol. 48, no. 3, pp. 702-711, Mar. 2000.

[6] R. Zhang, N. D. Sidiropoulos, and M. K. Tsatsanis, "Collision resolution in packet radio networks using rotational invariance techniques," IEEE Trans. Commun., vol. 50, no. 1, pp. 146-155, Jan. 2002.

[7] R. Lin and A. P. Petropulu, "A new wireless network medium access protocol based on cooperation," IEEE Trans. Signal Process., vol. 53, no. 12, pp. 4675-4684, Dec. 2005.

[8] H. Yang, A. P. Petropulu, X. Yang, and T. Camp, "A novel location relay selection scheme for ALLIANCES," IEEE Trans. Veh. Technol., vol. 57, no. 2, pp. 1272-1284, Mar. 2008.

[9] L. Dong and A. P. Petropulu, "Cooperative wireless medium access with multi-beam adaptive arrays," in Proc. IEEE Int. Workshop Signal Processing Advances Wireless Commun., 2005, pp. 870-874.

[10] X. Li, Y. Zhang, and M. G. Amin, "Cooperative wireless medium access exploiting multi-beam adaptive arrays and relay selection," presented at the IEEE Int. Conf. Circuits Systems Commun., Shanghai, China, May 2008.

[11] R. O. Schmidt, "Multiple emitter location and signal parameter estimation," IEEE Trans. Antennas Propag., vol. AP-34, no. 3, pp. 276-280, Mar. 1986

[12] R. Roy and T. Kailath, "ESPRIT-estimation of signal parameters via rotational invariance techniques," IEEE Trans. Acoust., Speech, Signal Process., vol. 37, no. 7, pp. 984-995, Jul. 1989.

[13] Y. Zhang, X. Li, and M. G. Amin, "Mobile ad hoc networks exploiting multi-beam antennas," in Handbook on Advancements in Smart Antenna Technologies for Wireless Networks, C. Sun, J. Cheng, and T. Ohira, Eds. Hershey, PA: IGI Global, 2008.

[14] X. Li, Y. Zhang, and M. G. Amin, "Node throughput analysis of decentralized wireless networks using multibeam antennas in multipath environments," Wireless Personal Commun., vol. 50, no. 4, pp. 447-456, Sep. 2008.

[15] X. Li, Y. Zhang, and M. G. Amin, "Multibeam antenna scheduling in ad hoc wireless networks," presented at the SPIE Symp. Defense Security Symp., Orlando, FL, Mar. 2008.

[16] X. Li, Y. Zhang, and M. G. Amin, "Performance of wireless networks exploiting multi-channel smart antennas in multipath environments," presented at the Int. Waveform Diversity Design Conf., Pisa, Italy, Jun. 2007.

[17] Y. Zhang, X. Li, and M. G. Amin, "Multi-channel smart antennas in wireless networks," presented at the Asilomar Conf. Signals, Systems Computers, Pacific Grove, CA, Oct. 2006.

[18] H. C. Lin, "Spatial correlations in adaptive arrays," IEEE Trans. Antennas Propag., vol. 30, no. 2, pp. 212-223, Mar. 1982.

[19] G. Dimic, N. D. Sidiropoulos, and L. Tassiulas, "Wireless networks with retransmission diversity access mechanisms: Stable throughput and delay properties," IEEE Trans. Signal Process., vol. 51, no. 8, pp. 2019-2030, Aug. 2003. 
[20] G. Dimic, N. D. Sidiropoulos, and R. Zhang, "Multiaccess queuing from a signal processing perspective-medium access control-physical cross-layer design,” IEEE Signal Process. Mag., vol. 21, no. 5, pp. 40-50, Sep. 2004.

[21] J. Shen, "On the singular values of Gaussian random matrices," Linear Algebra Appl., vol. 326, no. 1-3, pp. 1-14, 2001.

[22] G. Burel, "Statistical analysis of the smallest singular value in MIMO transmission systems," presented at the Int. Conf. on Signal, Speech, Image Processing, Skiathos, Greece, Sep. 2002.

[23] A. Grant, "Rayleigh fading multi-antenna channels," EURASIP J. Appl. Signal Process., no. 3, pp. 316-329, 2002.

[24] R. K. Mallik, "The pseudo-Wishart distribution and its application to MIMO systems," IEEE Trans. Inf. Theory, vol. 49, no. 10, pp. 2761-2769, Oct. 2003.

[25] T. Ratnarajah, R. Vaillancourt, and M. Alvo, "Eigenvalues and condition numbers of complex random matrices," SIAM J. Matrix Anal. Appl., vol. 26, no. 2, pp. 431-456, 2005.

[26] A. T. James, "Distributions of matrix variate and latent roots derived from normal samples," Ann. Math. Stat., vol. 35, no. 2, pp. 475-501, Jun. 1964.

[27] R. Kwan, C. Leung, and P. Ho, "Distribution of ordered eigenvalues of Wishart matrices," Electron. Lett., vol. 43, no. 5, Mar. 2007.

[28] Y. R. Zheng and C. Xiao, "Improved models for the generation of multiple uncorrelated Rayleigh fading waveforms," IEEE Commun. Lett., vol. 6, no. 6, pp. 256-258, Jun. 2002.

[29] X. Li and Y. Zhang, "Throughput analysis of cooperative wireless medium access scheme exploiting multi-beam adaptive arrays," presented at the IEEE Int. Conf. Acoustics, Speech, Signal Processing, Taipei, Taiwan, R.O.C., Apr. 2009.

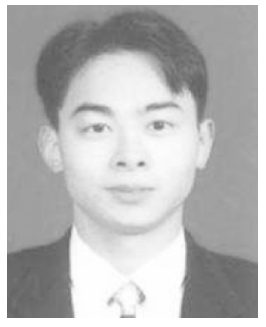

Xin Li (M'08) received the B.S. degree in electrical engineering, and M.S. degree in information and communications engineering from Chongqing University of Posts and Telecommunications (CQUPT), Chongqing, China, in 1999 and 2002, respectively, and the Ph.D. degree in information and communications engineering (as well as electromagnetic theory and microwave technology) from the University of Electronic Science and Technology of China (UESTC) in 2005

From December 1999 to May 2001, he was a Research Engineer with Datang Technology Company, Ltd., Beijing, China, where he accomplished his Master's thesis work and participated in drafting and amending the $3 \mathrm{G}$ time-division synchronous code-division multiple access (TD-SCDMA) standard and in prototyping the TD-SCDMA/GSM system. From January to July of 2002, he was a Hardware (RF) Research Engineer in Holley Company, Ltd., where he developed the dual-mode cell phone in cooperation with the Research adn Development Department in Dallas, TX. From September 2002 to June 2005, he was a Ph.D. candidate and developed a multiple-input-multiple-output-orthogonal frequency-division multiplexing (MIMO-OFDM) system and carried out extensive channel measurement campaign. From July 2005 to August 2006, he was a Research Scientist with the Alcatel Shanghai Bell Research \& Innovation Center, where he accomplished his postdoctoral research and focused on prototyping the MIMO-OFDM system for future $4 \mathrm{G}$ systems and on other projects such as the radio-over-fiber and distributed antenna system. Since September 2006, he has been with Villanova University, Villanova, PA, where he is currently a Postdoctoral Research Fellow with the Center for Advanced Communications. He is an active worldwide paper/book/project reviewer. He has published over 50 papers. His research interests include wireless channel modeling, source localization and tracking, cooperative communications, advanced signal processing and applications, communication network, ad hoc and mesh networks, radio frequency identification (RFID), and circuit and antenna design.

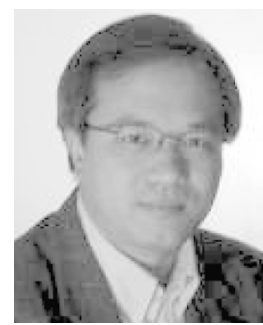

Yimin Zhang (SM'01) received the Ph.D. degree from the University of Tsukuba, Tsukuba, Japan, in 1988. He joined the faculty of the Department of Radio Engineering, Southeast University, Nanjing, China, in 1988. He served as a Technical Manager at the Communication Laboratory Japan, Kawasaki, Japan, from 1995 to 1997, and was a Visiting Researcher at ATR Adaptive Communications Research Laboratories, Kyoto, Japan, from 1997 to 1998. Since 1998, he has been with the Villanova University, Villanova, PA, where he is currently a Research Professor with the Center for Advanced Communications. His research interests lie in the area of statistical signal and array processing for communications and radar applications, including digital mobile communications, wireless networks, MIMO systems, cooperative communications, blind signal processing, jammer suppression, time-frequency analysis, source localization and target tracking, radio frequency identification (RFID), and image processing.

Dr. Zhang is an Associate Editor for the Journal of the Franklin Institute and serves on the editorial board of the Signal Processing journal. He is an Associate Editor for the IEEE SignAL PROCESSING LETTERS.

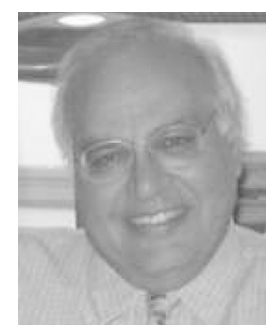

Moeness G. Amin (F'00) received his Ph.D. degree from the University of Colorado, Boulder, in 1984

He has been on the Faculty of Villanova University, Villanova, PA, since 1985, where is now a Professor in the Department of Electrical and Computer Engineering and the Director of the Center for Advanced Communications. He has over 400 publications in the areas of wireless communications, time-frequency analysis, smart antennas, interference cancellation in broadband communication platforms, direction finding, GPS technologies, over-the-horizon radar, and radar imaging.

Dr. Amin is the recipient of the 2009 Individual Technical Achievement Award from the European Association of Signal Processing. He is a Fellow of the International Society of Optical Engineering; recipient of the IEEE Third Millennium Medal; Distinguished Lecturer of the IEEE Signal Processing Society for 2003 and 2004; Member of the Franklin Institute Committee on Science and the Arts; recipient of the 1997 Villanova University Outstanding Faculty Research Award; and recipient of the 1997 IEEE Philadelphia Section Service Award. He was a Guest Editor of the Journal of Franklin Institute September 2008 Special Issue on Advances in Indoor Radar Imaging. He is a Guest Editor of the IEEE TRANSACTIONS ON GEOSCIENCE AND REMOTE SENSING May 2009 Special issue on Remote Sensing of Building Interior. He is the Co-Guest Editor of IET Signal Processing upcoming Special Issue on Time-Frequency Approach to Radar Detection, Imaging, and Classification. $\mathrm{He}$ was the Co-Chair of the Special Sessions of the 2008 IEEE International Conference on Acoustics, Speech, and Signal Processing, Nevada. He was the Technical Chair of the Second IEEE International Symposium on Signal Processing and Information Technology, Morocco, 2002; the General and Organization Chair of the IEEE Workshop on Statistical Signal and Array Processing, Pennsylvania, 2000; and the General and Organization Chair of the IEEE International Symposium on Time-Frequency and Time-Scale Analysis, Pennsylvania, 1994. He was an Associate Editor of the IEEE TRANSACTIONS ON SigNAL PROCESSING from 1996 to 1998. He was a member of the IEEE Signal Processing Society Technical Committee on Signal Processing for Communications from 1998 to 2002 and was a Member of the IEEE Signal Processing Society Technical Committee on Statistical Signal and Array Processing from 1995 to 1997. 